\title{
Secondary Augmentation Mastopexy to Correct Malpositioned Nipple Areola Complex (NAC) and Baker's Grade III Capsular Contracture 15 Years Later
}

Francesco Mazzarone, Lee Seng Khoo* and Luiggi Fayad

Ivo Pitanguy Institute, Rua Dona Mariana, 65 - Botafogo, Rio de Janeiro, RJ, Brasil

"Corresponding author: Khoo LS, Ivo Pitanguy Institute, Rua Dona Mariana, 65 - Botafogo, Rio de Janeiro, RJ, Brasil, Tel: ; Fax: (21) 2539-0314; E-mail: khooleeseng@hotmail.com

Received date: May 05, 2015, Accepted date: June 28, 2015, Published date: June 30, 2015

Copyright: (c) 2015 Mazzarone et al. This is an open-access article distributed under the terms of the Creative Commons Attribution License, which permits unrestricted use, distribution, and reproduction in any medium, provided the original author and source are credited.

\begin{abstract}
We report a case of a 51 year old woman who underwent a no vertical scar breast reduction with augmentation mastopexy 15 years ago and presented with breast distortion, gross asymmetry with medialized nipples and Baker's Grade III capsular contracture. This report details the surgical steps undertaken to correct the deformity and the postoperative results. Secondary augmentation mastopexy presents a unique challenge due to the limited amount of tissue to work with and decreased blood supply to the flaps.
\end{abstract}

\section{Level of Evidence V}

\section{Introduction}

Augmentation mastopexy is a complex procedure that combines two surgical procedures in a single operation. Augmentation mastopexy attempts to correct both mammary volume loss or depletion and ptosis. Some surgeons have suggested performing both procedures in a separate setting- performing the mastopexy first if the primary problem is one of ptosis and performing augmentation mammaplasty as a second procedure $[1,2]$. If the primary problem is of volume loss or deflation, augmentation mammaplasty is carried out first followed by mastopexy in another setting. Splitting the surgery into two stages have both economical and social drawbacks to the patient and surgeon. The patient now has to pay for and undergo two separate surgeries as well as be subjected to two separate postoperative recovery periods.

Augmentation mastopexy does have its advantages that it allows the surgeon to pick an appropriate implant volume for the breast while accurately determining how much skin or tissue needs to be resected thereafter [3-5]. However, the complication rates for augmentation mastopexy are arbritarily higher than two stage procedures [2,6-9]. Secondary or reoperative augmentation mastopexy presents a unique challenge due to the limited amount of tissue to work with and decreased blood supply to the flaps.

We present a case report of a 51 year old woman who underwent augmentation mastopexy 15 years ago and presented with breast distortion, gross asymmetry with medialized nipples and Baker's Grade III capsular contracture. This report details the preoperative condition and the operative steps undertaken to correct the deformity. The postoperative result and patient satisfaction are also discussed.

\section{Case report}

A 51 year old lady presented to our practice complaining of breast asymmetry, distortion and malpositioned nipples (Figures 1-3). She underwent augmentation mastopexy at another practice 15 years ago and has been unhappy with the postoperative results ever since. She finally decided to seek corrective surgery almost a decade and a half later as she feels her breasts are becoming harder and more contorted.

Surgical history revealed that she had $90 \mathrm{cc}$ bilateral silicone implants placed in the subglandular plane via a no vertical scar breast reduction and mastopexy technique. The surgeon performed a doughnut mastopexy and resected tissue and skin via an inframammary incision that extended all the way laterally. On examination, we found that the patient had distorted and asymmetrical breasts. The nipple areola complex (NAC) was too medialized and the position was asymmetrical on both sides. The NAC was mildly stretched out and widened. The subglandular pocket has also been dissected too medially on the lower poles of the pocket. The breasts were firm, hard and deformed but were not painful to palpation. We assigned a Baker's Grade III capsular contracture to the condition (Figures 1-3).

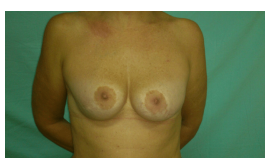

Figure 1: Frontal view pre-op.

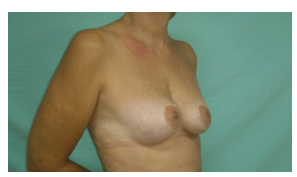

Figure 2: Right 3/4 view pre-op. 
Page 2 of 4

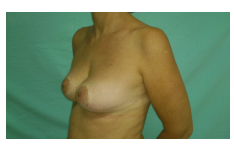

Figure 3: Left 3/4 view pre-op.

The patient reiterated that what bothered her most was the grossly medialized nipples and that the right nipple was also higher than the left. We counseled the patient that a tradeoff will have to be made on a horizontal or vertical scar component in redo augmentation mastopexy in an attempt to medialize the NAC. She accepted the tradeoff and also requested for more fill in the breasts of which we decided to opt for larger implants.

During surgery, we marked the optimal position for the new nipple and outlined the circumareolar "doughnut mastopexy" incision (Figure 4).

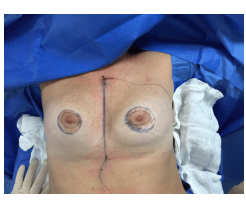

Figure 4: Marking of doughnut mastopexy around nipple areolar complex (NAC).

The position and symmetry was rechecked with the triangulation technique using two sutures- one placed in the sternal notch and another at the xiphoid.

The Schwartzman procedure was carried out and skin excised leaving dermis. An incision was made in the lower border of the dermis immediately caudal to the NAC. In order to maintain adequate tissue thickness between skin and implant capsule, the incision was beveled. Dissection was carried down until the capsules of the implants were seen. At this stage an incision was made into the capsule and the previous implants were removed (Figure 5).

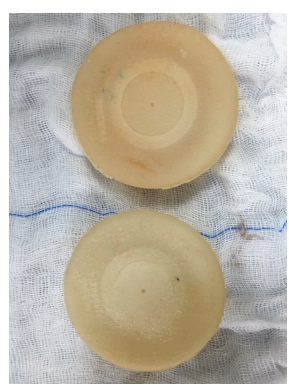

Figure 5: Previous implants removed - 90cc round low profile textured implants.

Capsulotomy was performed but the capsule was left in to provide additional coverage for soft tissue and not to further disrupt blood supply. Adequate but not excessive pocket dissection was carried out in the subglandular plane. The procedure was repeated on the contralateral side.

After adequate hemostasis and pocket washout; $300 \mathrm{cc}$ HI profile polyurethrane coated highly cohesive silicone implants (soaked in a solution of $1.5 \mathrm{~g}$ Cefuroxime and $80 \mathrm{mg}$ of Gentamicin) were inserted into the breast pockets bilaterally. However the NAC was still too medial in relation to the mammary gland. The NAC was pushed laterally and fusiform ellipse of skin medial to the NAC was marked in methylene blue and excised. This allowed the NAC to be displaced laterally obtaining a more aesthetic appearance (Figure 6).

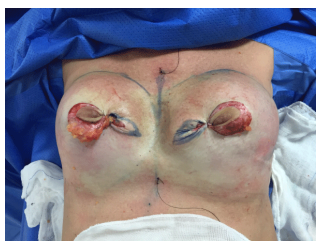

Figure 6: Fusiform skin ellipse marked to be excised and NAC placed laterally.

Due to the vector and position of the NAC relative to the desired degree of repositioning of the NAC, the final scars lie in the horizontal position on the right and in a transverse position on the left (Figure 7).

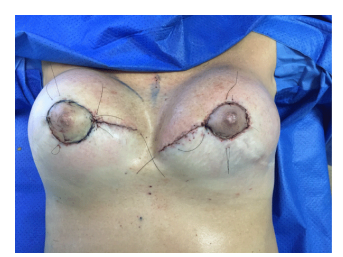

Figure 7: Immediate post-operative result (frontal view).

The tissues were closed in layers utilizing buried interrupted 3-0 Nylon for deep tissue, 4-0 buried interrupted Nylon for dermal layer and subcuticular 4-0 Nylon for intradermal closure. The areolar was closed with 4-0 Nylon in the subdermal plane and subcuticular 4-0 Nylon for intradermal closure. The immediate post operative result is seen here (Figures 7 and 8).

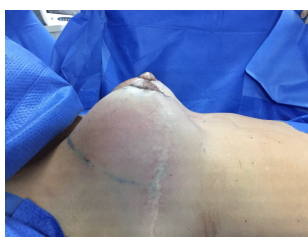

Figure 8: Immediate post-operative result (lateral view).

There was good medialization of the NAC and restoration of volume to the of breasts. The breasts have a rounder more aesthetic shape with the tradeoff being the scars in the medial region of the breast (Figure 9-11). 


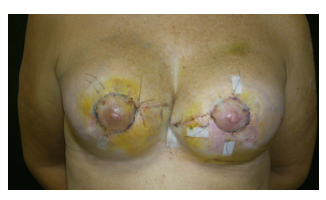

Figure 9: Frontal view Day 10 post-op.

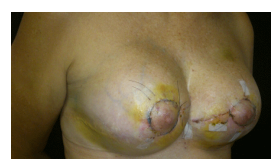

Figure 10: Right 3/4 Day 10 view post-op.

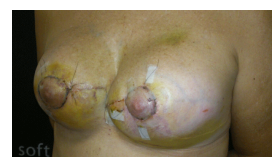

Figure 11: Left 3/4 Day 10 view post op.

However, the recovery period was complicated by wound dehiscence surrounding the NAC bilaterally being more prominent on lower quadrant of the left side. The dehiscence was treated conservatively with hyrofiber wound dressing with ionic silver. The wounds were allowed to heal by secondary intention. Although the dehiscence compromised the end aesthetic result, there was marked improvement in the breast form. The patient has been counseled on minor revisional surgery but is happy and satisfied with the current appearance of her breasts (Figures 12-15).

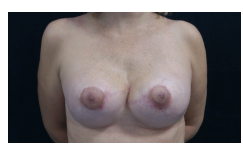

Figure 12: Frontal view 8 weeks post-op.

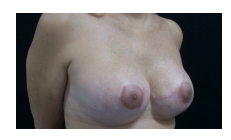

Figure 13: Right 3/4 view 8 weeks post-op.

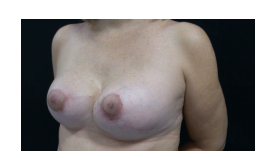

Figure 14: Left 3/4 view 8 weeks post-op.

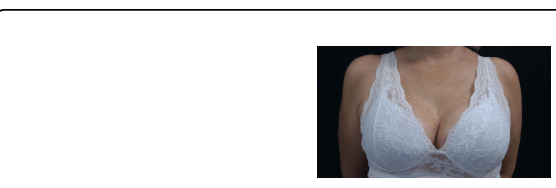

Figure 15: Frontal view 8 weeks post-op fully clothed.

\section{Discussion}

Gonzalez-Ulloa and Regnault [4,5] were the first to conceive the idea of combining an implant with a lift to correct hypoplastic and ptotic breasts in the 1960s. Since then,there has been a plethora of published material for and against augmentation mastopexy as a single stage surgery.

Proponents cite that augmentation mastopexy provides the surgeon with the means to address the ptotic and hypoplastic breast in one single surgery while being able to determine the ideal shape, symmetry and position of the nipple areolar complex (NAC) $[3,4,5]$. Critics state that the goals of augmentation and mastopexy are opposite [1]. Augmentation seeks to expand volume (and inadvertently skin/soft tissues) while mastopexy attempts to eliminate the redundant skin/ subcutaneous tissue thereby lifting the breast [1]. Augmentation mastopexy may therefore augment the risk of complications such as hypertrophic scars, compromised healing, infection, capsular contracture and implant extrusion [2,6-9].

Cardenas-Camarena reported a complication rate of $18 \%$ based on 384 patients undergoing one stage mastopexy-augmentation. He described the complications as minor and eschews the benefits of the one stage procedure [10].

Stevens in his publication of 321 consecutive one stage augmentation mastopexy of which two thirds were primary cases and the remainder secondary cases - reported a revision rate of $14.6 \%$. Stevens states that these results were acceptable to his $13 \%$ revision rate breast implant only patients and an $8.6 \%$ revision rate in his mastopexy only patients [11].

Spear on the other hand noted a 10 -fold increase in complications comparing primary augmentation mastopexy with primary augmentation only cases [2]. Tebbetts recommends splitting the surgery to two stages to avoid any increased risk of complications associated with augmentation mastopexy. Tebbetts states that a fundamental problem with mastopexy augmentation is that the goals and objectives of each operation contradict the goals and objectives of the other [1].

It is imperative to note that secondary augmentation mastopexy carries an even higher risk of complications and patient dissatisfaction. Handel in his publication 'Secondary mastopexy in the augmented patient: a recipe for disaster' outlines the difficulties and complications that follows reoperative augmentation mastopexy. Augmented breasts become ptotic overtime and the pressure exerted by implants also contribute to thinning of the skin and soft tissues of the breast. In the majority of cases, these effects are seen in the lower pole of the breast. There is also a high degree of capsular contracture that further complicates the managements. Management may require explantation only, mastopexy with explantation or mastopexy with or without implant exchange or pocket change [12]. 
Citation: Mazzarone F, Fayad L, Khoo LS (2015) Secondary Augmentation Mastopexy to Correct Malpositioned Nipple Areola Complex (NAC) and Baker's Grade III Capsular Contracture 15 Years Later. Anaplastology 4: 149. doi:10.4172/2161-1173.1000149

Page 4 of 4

Preoperatively the surgeon has to identify all scars surrounding the breast particularly if the surgery was carried out elsewhere and no intraoperative details are available. The scars directly relate to the vascular supply of the NAC and gives the surgeon valuable information on the ideal pedicle of choice. Inferior pole breast tissues often have disrupted blood supply due to previous incisions and excisions. It is safer to select a superior pedicle where breast tissue blood supply is often better and undisrupted [13].

When performing circumareolar "doughnut" incisions when placing a subglandular implant such as this case, it is imperative to bevel the plane of dissection acutely due to the thin nature of the tissue between the skin and the underlying implant. Failure to execute this extremely important step will result in impaired blood supply to the $\mathrm{NAC}$ and possible necrosis [13]. We also recommend performing only capsulotomy and not capsulectomy as the bleeding and edema that ensues may increase the chances of an early recurrent capsular contracture. The retained capsule has ingrown blood supply that ideally should be left intact.

In conclusion, secondary augmentation mastopexy is a complex procedure that carries with it a high degree of complication and patient dissatisfaction. Although one stage augmentation mastopexy carries its advantages, we recommend exercising caution in patient selection and when in doubt, to perform the surgery as a two stage procedure. In secondary cases, a good patient doctor rapport must be established and potential side effects and complications of a revision must be explained in depth to afford a realistic expectation on the patient's behalf.

Conflict of Interest: The authors have no conflict of interests to declare.

\section{References}

1. Tebbetts JB (2008) Augmentation mammoplasty redefining the patient and surgeon experience. Chapter 20 -Augmentation mastopexy. Edinburgh, Mosby
2. Spear S (2003) Augmentation/Mastopexy: "Surgeon, Beware". Plast Reconstr Surg 112: 905-906.

3. de la Fuente A, Martín del Yerro JL (1992) Periareolar mastopexy with mammary implants. Aesthetic Plast Surg 16: 337-341.

4. Elliott LF (2002) Circumareolar mastopexy with augmentation. Clin Plast Surg 29: 337-347.

5. Karnes J, Morrison W, Salisbury M, Schaeferle M, Beckham P, et al. (2000) Simultaneous breast augmentation and lift. Aesthetic Plast Surg 24: 148-154.

6. Spear SL, Pelletiere CV, Menon N (2004) One-stage augmentation combined with mastopexy: aesthetic results and patient satisfaction. Aesthetic Plast Surg 28: 259-267.

7. Spear SL, Low M, Ducic I (2003) Revision augmentation mastopexy: indications, operations, and outcomes. Ann Plast Surg 51: 540-546.

8. Baran CN, Peker F, Ortak T, Sensoz O, Baran NK (2001) Unsatisfactory results of periareolar mastopexy with or without augmentation and reduction mammoplasty: enlarged areola with $\mathrm{fl}$ attened nipple. Aesthetic Plast Surg 25: 286-289

9. Persoff MM (2003) Vertical mastopexy with expansion augmentation. Aesthetic Plast Surg 27: 13-19.

10. Cárdenas-Camarena L, Ramírez-Macías R; International Confederation for Plastic Reconstructive and Aesthetic Surgery; International Society of Aesthetic Plastic Surgery; Iberolatinoamerican Plastic Surgery Federation; Mexican Association of Plastic Esthetic and Reconstructive Surgery; Western Mexican Association of Plastic, Esthetic and Reconstructive Surgery; Jalisco College of Plastic Surgeons (2006) Augmentation/mastopexy: how to select and perform the proper technique. Aesthetic Plast Surg 30: 21-33.

11. Stevens WG, Freeman ME, Stoker DA, Quardt SM, Cohen R, et al. (2007) One-stage mastopexy with breast augmentation: a review of 321 patients. Plast Reconstr Surg 120: 1674-1679.

12. Handel N (2006) Secondary mastopexy in the augmented patient: a recipe for disaster. Plast Reconstr Surg 118: 152S-163S.

13. Shiffman, Melvin A (2009) Mastopexy and Breast Reduction: Principles and Practice. Chapter 21 -Breast Augmentation and Mastopexy: How to Select and Perform the Techniques Minimizing Complications by Lázaro Cárdenas-Camarena. pp 163-178. Berlin, Heidelberg. 